\title{
SEMILEPTONIC B DECAYS IN BABAR
}

\author{
A.Sarti ${ }^{a}$ \\ Dep. of Physics, The University of Ferrara and INFN, I-44100 Ferrara, Italy
}

\begin{abstract}
BABAR measurements involving semileptonic decays of $B$ mesons are reviewed. Attention is focused on the extraction of $\left|V_{u b}\right|$ and $\left|V_{c b}\right|$ elements of the Cabibbo-KobayashiMaskawa quark mixing matrix. Recent results of inclusive and exclusive approaches are presented.
\end{abstract}

The study of semileptonic $B$ decays provides observables for the extraction of $\left|V_{u b}\right|$ and $\left|V_{c b}\right|$ elements of Cabibbo-Kobayashi-Maskawa matrix, the measurement of the $b$-quark mass and the extraction of non-perturbative QCD parameters $\left(\bar{\Lambda}, \lambda_{1}\right)$. The theoretical framework for such measurements consists mainly of two different approaches: the Operator Product Expansion (OPE)[1], used for the extraction of inclusive semileptonic rates, and the Heavy Quark Effective Theory, providing the tools for handling exclusive decays. Both approaches need corrections when used in real measurements: the analysis cuts, used to reject background events, are reducing the decay kinematic phase space and thus have to be included in the OPE. The uncertainty on those corrections is currently giving the higher contribution to the systematic error. Similarly, in $\left|V_{c b}\right|$ exclusive measurements, an extrapolation to a phase space boundary is needed when using HQET.

From the experimental point of view the reconstruction of a large $B$ meson sample $\left(\mathrm{BR}(b \rightarrow u \ell v) \sim 10^{-3}\right)$ and an accurate reconstruction of the $B$ decay chain (the current relative error on $\left|V_{c b}\right|$ is $\sim 2 \%$ ) are main issues. A dedicated $B$ meson reconstruction technique has been set up in BABAR to fulfill the following requirements. A large sample of $B$ mesons can be collected by selecting hadronic decays $B_{\text {reco }} \rightarrow D^{(*)} X$, where $X$ represents a collection of hadrons composed of $\pi, K, K_{s}^{0}, \pi^{0}$ mesons. The kinematic consistency of $B_{\text {reco }}$ candidates is checked with two variables, the beam energysubstituted mass $m_{E S}=\sqrt{s / 4-\vec{p}_{B}^{2}}$ and the energy difference $\Delta E=E_{B}-\sqrt{s} / 2$. Here $\sqrt{s}$ is the total energy in the $\Upsilon(4 S)$ center of mass frame, and $\vec{p}_{B}$ and $E_{B}$ denote both the momentum and energy of the $B_{\text {reco }}$ candidate, in the same frame. The advantages of fully reconstructing one $B$ meson in the event are: an easier subtraction of the continuum background, the knowledge of decay kinematics of the other $B$ (the only missing particle should be the neutrino) and the possibility to request flavor and charge correlations between the fully reconstructed $B$ meson and the one decaying semileptonically $\left(B_{\text {recoil }}\right)$. On the recoil side, semileptonic events can be selected using a cut on the lepton momentum $(\sim 0.5 \div 1 \mathrm{GeV} / c)$ and on the neutrino four-momentum. The efficiency of this reconstruction technique is $\sim 0.1 \div 0.4 \%$. 

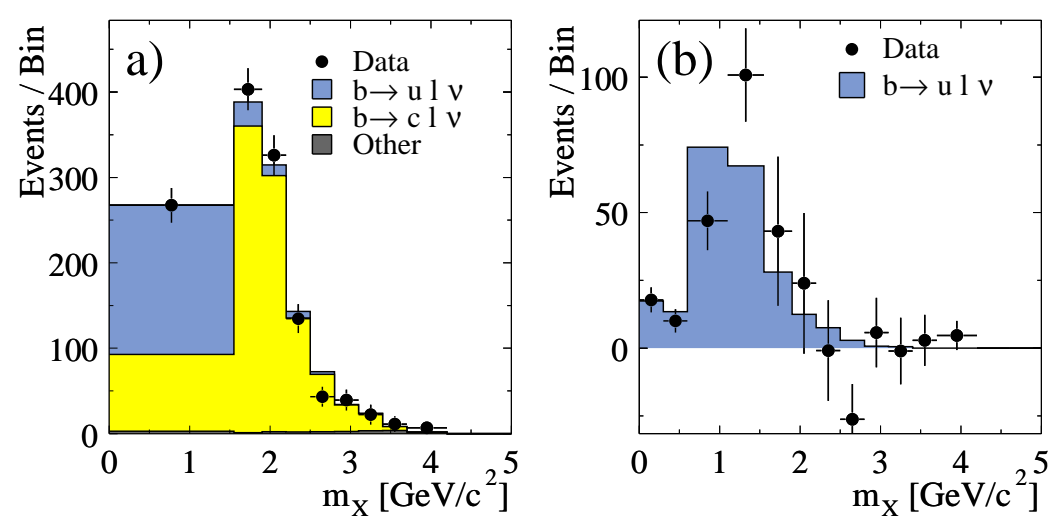

Figure 1: The $m_{X}$ distribution for $\bar{B} \rightarrow X \ell \bar{v}$ candidates for BABAR inclusive analysis: a) data (points) and fit components, and b) data and signal MC after subtraction of the $b \rightarrow c \ell v$ and the "other" backgrounds.

\section{Inclusive $\left|V_{u b}\right|$ measurement}

The main experimental issue of this analysis is the rejection of the huge $\bar{B} \rightarrow X_{c} \ell \bar{v}^{b}$ background $\left(\mathcal{B}\left(\bar{B} \rightarrow X_{c} \ell \bar{v}\right) \sim 60 \times \mathcal{B}\left(\bar{B} \rightarrow X_{u} \ell \bar{v}\right)\right)$. The $B_{\text {recoil }}$ charmless semileptonic decays are selected with a cut on the lepton momentum, a cut on the neutrino mass and a veto on neutral and charged kaons. The residual background is determined from a fit of the hadronic invariant mass $\left(m_{X}\right)$ distribution and $\left|V_{u b}\right|$ is extracted from the measured semileptonic rate using the OPE relation $|2|:\left|V_{u b}\right|=0.00445 \sqrt{\left(\frac{B(b \rightarrow u \ell v) \cdot 1.55 p s}{0.002 \cdot \tau_{B}}\right)} \times$ $\left(1.0 \pm 0.020_{\text {pert }} \pm 0.052_{1 / \mathrm{m}_{b}^{3}}\right)$. In order to reduce systematic uncertainties, the ratio of branching ratios $R_{u / s l}=\mathcal{B}\left(\bar{B} \rightarrow X_{u} \ell \bar{v}\right) / \mathcal{B}(\bar{B} \rightarrow X \ell \bar{v})$ is determined from $N_{u}$, the observed number of $\bar{B} \rightarrow X_{u} \ell \bar{v}$ candidates with $m_{X}<1.55 \mathrm{GeV} / c^{2}$, and $N_{s l}$, the number of events with at least one charged lepton: $R_{u / s l}=\frac{N_{u} /\left(\varepsilon_{s e l}^{u} \varepsilon_{T_{X}}^{u}\right)}{\left.N_{s l}\right)} \times \frac{\varepsilon_{l}^{s l} \varepsilon_{r e c o}^{s l}}{\varepsilon_{l}^{u} \varepsilon_{\text {reco }}^{u}}$. Here $\varepsilon_{s e l}^{u}$ is the efficiency for selecting $\bar{B} \rightarrow X_{u} \ell \bar{v}$ decays once a $\bar{B} \rightarrow X \ell \bar{v}$ candidate has been identified, $\varepsilon_{m_{X}}^{u}$ is the fraction of signal events with $m_{X}<1.55 \mathrm{GeV} / c^{2}, \varepsilon_{l}^{s l} / \varepsilon_{l}^{u}$ corrects for the difference in the efficiency of the lepton momentum cut for $\bar{B} \rightarrow X \ell \bar{v}$ and $\bar{B} \rightarrow X_{u} \ell \bar{v}$ decays, and $\varepsilon_{\text {reco }}^{s l} / \varepsilon_{\text {reco }}^{u}$ accounts for a possible efficiency difference in the $B_{\text {reco }}$ reconstruction in events with $\bar{B} \rightarrow X \ell \bar{v}$ and $\bar{B} \rightarrow X_{u} \ell \bar{v}$ decays. $N_{s l}$ is derived from a fit to the $m_{\mathrm{ES}}$ distribution. $N_{u}$ is extracted from the $m_{X}$ distribution by a minimum $\chi^{2}$ fit to the sum of three contributions: the signal, the background $N_{c}$ from $\bar{B} \rightarrow X_{c} \ell \bar{\nu}$, and a background of $<1 \%$ from other sources (misidentified leptons, secondary $\tau$ and charm decays). Fig. 11 shows the fitted $m_{X}$ distribution. Fig. 1b shows the $m_{X}$ dis-

a e-mail: asarti@slac.stanford.edu

${ }^{b}$ Charge conjugation is implied. 

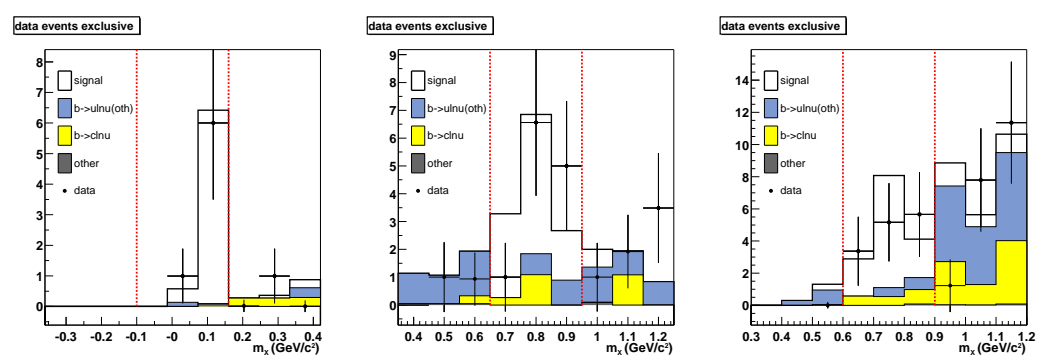

Figure 2: Projections in the $m_{X}$ variable for the BABAR $\left|V_{u b}\right|$ exclusive analysis. Vertical dotted lines represent the signal region. Left: $B^{ \pm} \rightarrow \pi^{0} l v$; middle: $B^{ \pm} \rightarrow \rho^{0} l v$; right: $B^{ \pm} \rightarrow \omega l v$.

tribution after background subtraction. By using $82 \mathrm{fb}^{-1}$ integrated luminosity on the $\Upsilon(4 S)$ peak, $B A B A R$ obtains $\left|V_{u b}\right|=(4.62 \pm 0.28 \pm 0.27 \pm 0.40 \pm 0.26) \times 10^{-3}$, where errors are respectively statistical, detector systematic, theoretical model and propagation of error from the OPE relation [2]. The signal over background ratio $(\mathrm{S} / \mathrm{B})$ is 1.7 (higher than any previous inclusive analysis) and the main error comes from the parametrization of the $b$-quark Fermi motion inside the $B$ meson parametrization.

Reduction of theoretical systematic error, related to the shape function parameterization of the Fermi motion, can be achieved by adding a cut on the di-lepton pair invariant mass $\left(q^{2}\right)$, to the $m_{X}$ one, which allows for a reduction of systematic error of $\sim 35 \%$ on $\left|V_{u b}\right|$.

\section{Exclusive $\left|V_{u b}\right|$ measurement}

Using the same $B$ meson reconstruction technique as in the inclusive analysis, and with a similar analysis strategy and signal events selection, it is possible to study the exclusive $\bar{B} \rightarrow X_{u} \ell \bar{v}$ decays. The high purity of the sample of reconstructed $B$ mesons makes possible to use the mass of the hadronic system $\left(m_{X}\right)$ to separate the resonances. $B^{ \pm} \rightarrow \pi^{0} l v, B^{ \pm} \rightarrow \rho^{0} l v$ and $B^{ \pm} \rightarrow \omega l v$ decays are selected applying constraints on the missing mass and $m_{X}$. The measured exclusive branching ratios are: $\mathcal{B}\left(B^{ \pm} \rightarrow \pi^{0} l v\right)=\left(0.78 \pm 0.32_{\text {stat }} \pm 0.13_{\text {syst }}\right) 10^{-4}, \mathcal{B}\left(B^{ \pm} \rightarrow \rho^{0} l v\right)=(0.99 \pm$ $\left.0.37_{\text {stat }} \pm 0.19_{\text {syst }}\right) 10^{-4}$ and $\mathcal{B}\left(B^{ \pm} \rightarrow \omega l v\right)=\left(2.20 \pm 0.92_{\text {stat }} \pm 0.57_{\text {syst }}\right) 10^{-4}$, where the main contribution to the systematic error comes from the uncertainty on signal $\mathrm{MC}$ modeling and the fit to the $m_{\mathrm{ES}}$ distributions used to extract the number of signal events. The $\mathcal{B}\left(B^{ \pm} \rightarrow \rho^{0} l v\right)$ result is obtained applying a cut on the two pions invariant mass $\left(0.65 \mathrm{GeV} / c^{2} \leq m_{\pi^{+} \pi^{-}} \leq 0.95 \mathrm{GeV} / c^{2}\right)$. In Figure 2 the projection of the results on the $m_{X}$ variable is shown. 

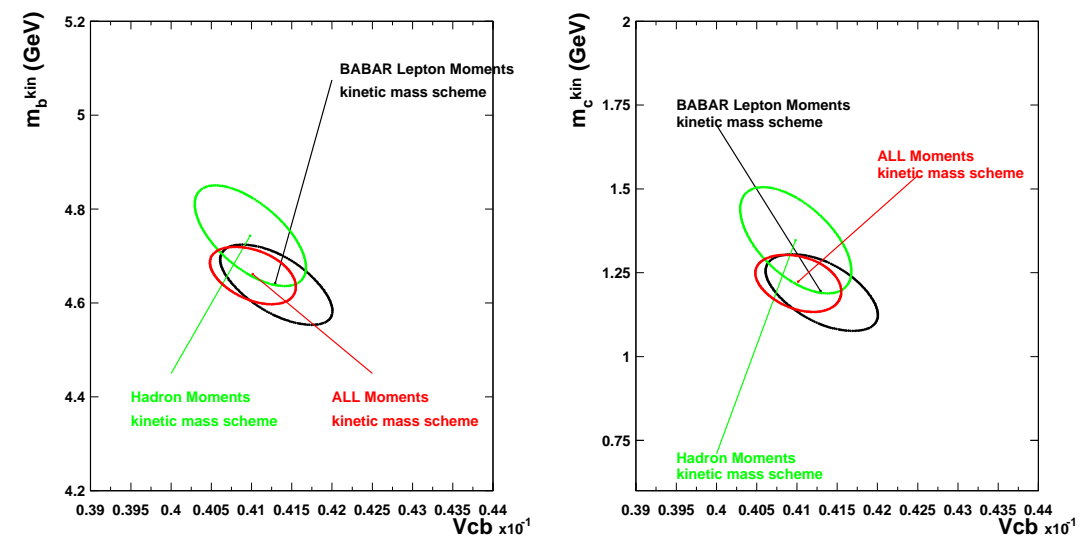

Figure 3: Fit results with contours corresponding to $\Delta \chi^{2}=1$ for (left) $\left|V_{c b}\right|$ versus $m_{b}$, and (right) $\left|V_{c b}\right|$ versus $m_{c}$, separately for fits using the hadron mass, the lepton energy, and all moments.

\section{Inclusive $\left|V_{c b}\right|$ measurement}

By measuring the first few moments $\left\langle m_{X}^{n}\right\rangle$ of the hadronic mass distributions in $\bar{B} \rightarrow X_{c} \ell^{-} \bar{v}$ decays, it is possible to constrain the OPE parameters and extract $\left|V_{c b}\right|$ and the heavy quark masses $m_{b}$ and $m_{c}$. The measurement of the lepton energy moments can also improve the determination of $\left|V_{c b}\right| 3 \mid . B$ mesons have been fully reconstructed with the technique discussed above. The semileptonic events selection proceeds using a cut on the lepton momentum and on the missing mass. Charge and flavor correlations are requested. The main sources of systematic errors are the precision in the modeling of the detector efficiency and particle reconstruction, the subtraction of the combinatorial background of the $B_{\text {reco }}$ sample, the residual background estimate and the uncertainties in the modeling of the hadronic states. The results do not depend on assumptions for branching fractions and mass distributions for higher mass hadronic states. Using a Heavy Quark Expansion in the kinetic mass scheme to order $1 / \mathrm{m}_{b}^{3}$, we extract the branching fraction, $\mathcal{B}_{c \ell v}=\left(10.62 \pm 0.16_{\text {exp }} \pm 0.06_{H Q E}\right) \%$, and the CKM matrix element, $\left|V_{c b}\right|=\left(41.25 \pm 0.45_{\text {exp }} \pm 0.41_{H Q E} \pm 0.62_{\text {theory }}\right) \times 10^{-3}$, with significantly reduced uncertainties. Figures 3 left) and 3 right) are showing the $\Delta \chi^{2}=1$ ellipses for $\left|V_{c b}\right|$ versus $m_{b}$ and $\left|V_{c b}\right|$ versus $m_{c}$ for the standard fit to all data and separate fits of the hadron and lepton moments, but including the truncated branching fractions in both.

\section{Exclusive $\left|V_{c b}\right|$ measurement}

A measurement of exclusively reconstructed $\bar{B}^{0} \rightarrow D^{*+} \ell^{-} \bar{v}_{\ell}$ decays rate $(\Gamma)$ can be used to extract $\left|V_{c b}\right|$ [4] by measuring the decay rate $d \Gamma / d w$ for $w>1$, where $w$ is 
the Lorentz boost of the $D^{*+}$ in the $\bar{B}^{0}$ rest frame, and extrapolating the rate to the kinematic limit corresponding to $w=1$. The analysis is based on a data sample of 79 (9.6) $\mathrm{fb}^{-1}$ recorded on (40 MeV below) the $\Upsilon(4 S)$ resonance. We select events containing a $D^{*}$ and an oppositely-charged electron or muon with momentum in the range $\left(1.2<p_{\ell}<2.4 \mathrm{GeV} / c\right)$. For each decay candidate we compute the cosine of the angle between momentum of the $\bar{B}^{0}$ and of the $D^{*+} \ell^{-}$pair, $\cos \theta_{B^{0}, D^{*} \ell}=\frac{2 E_{B^{0}} E_{D^{*}} \ell_{B^{0}}^{2}-M_{D^{*} \ell}^{2}}{2 p_{B^{0}} D_{D^{*} \ell}}$, that we fit in the range $-10<\cos \theta_{B^{0}, D^{*} \ell}<5$ to determine the signal contribution and the normalization of the uncorrelated and $B \rightarrow D^{*+} \pi \ell^{-} \bar{v}_{\ell}$ backgrounds. To extract $\left|V_{c b}\right|$, we compare the signal yields to the expected differential decay rate $\frac{\mathrm{d} \Gamma}{\mathrm{d} w}=\frac{G_{F}^{2}}{48 \pi^{3}} \mathcal{G}(w)\left[\left|V_{c b}\right| \mathcal{H}(w)\right]^{2}$, where $\mathcal{G}(w)$ is a known phase space factor and $\mathcal{H}(w)$ is the form factor. We consider two different parameterization of $\mathcal{H}(w)$. A simple Taylor expansion with three parameters (the extrapolation $\mathcal{F}(w=1)\left|V_{c b}\right|$, the slope $\rho_{\mathcal{F}}^{2}$, and the curvature $c$ ) and a parameterization with two parameters (the extrapolation $\left|V_{c b}\right| \mathcal{A}_{1}(w=1)$ and the slope $\left.\rho_{\mathcal{A}_{1}}^{2}\right)$. The two functions have different slopes, but in the limit $w \rightarrow 1$, we expect $\mathcal{A}_{1}(1)=\mathcal{F}(1)$. We perform a least-squares fit of the sum of the observed signal plus background yields to the expected yield in ten bins of $w$. Figure 4 compares the observed yield of signal and background events summed over all data samples with the result of the fit and illustrates the extrapolation to $w=1$ for the two form factor parameterizations. A major source of uncertainty is the reconstruction efficiency of the low-momentum pion from the $D^{*+}$ decay. Furthermore, there are several uncertainties related to the form factors and their parameterization. The fit results for the two different parameterizations of the dependence of the form factors on $w$ give consistent results. We adopt the result based on the more recent parameterization by Caprini et al. [5] and assign the observed difference in the extrapolation to $w \rightarrow 1$ as an additional systematic error. Using the recent lattice calculation [4], we obtain $\left|V_{c b}\right|=\left(38.03 \pm 0.68 \pm 1.07_{-1.15}^{+1.25}{ }_{-1.25}^{+1.45}\right) \times 10^{-3}$, where the first error is statistical, the second systematic, the third the model uncertainty (including the choice of the form factor expansion) and the fourth reflects the uncertainty in $\mathcal{A}_{1}(1)$.

\section{Conclusions and outlook}

$B A B A R$ studies of semileptonic $B$ decays have given a significant contribution to the understanding of the theoretical framework used to describe $b \rightarrow u$ and $b \rightarrow c$ transitions (OPE and HQET) and resulted in a consistent reduction of the error on the $\left|V_{u b}\right|$ and $\left|V_{c b}\right|$ CKM matrix elements. The $\left|V_{u b}\right|$ inclusive analysis result is currently the best single measurement with a relative error on $\left|V_{u b}\right|$ of $\sim 14 \%$ and $\mathrm{S} / \mathrm{B} \sim 1.7$ while the exclusive analysis gave promising preliminary results. The $\left|V_{c b}\right|$ exclusive analysis is currently the most precise single measurement and is consistent with results from Belle and LEP, while the preliminary result of the moment analysis will soon become public still reducing the uncertainty on $\left|V_{c b}\right|$.

${ }^{c}$ Momenta are measured in the $\Upsilon(4 S)$ rest frame, unless explicitely stated otherwise. 

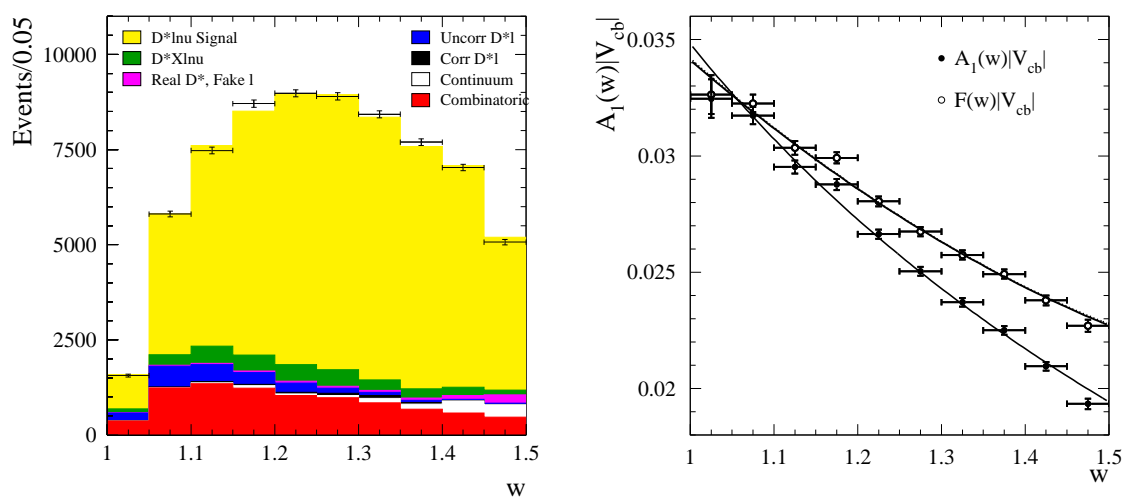

Figure 4: Left: Result of the fit (histograms) to the observed $w$ distribution (data points). Right: Form factor dependence on $w$, fits (lines) and data corrected for background and efficiency, all the terms in the decay rate except for $\left|V_{c b}\right| \mathcal{F}(w)$ for two different parameterizations (see text for details).

\section{References}

[1] I. I. Y. Bigi, M. A. Shifman, N. G. Uraltsev, and A. I. Vainshtein, Phys. Rev. Lett. 71, 496 (1993); A. V. Manohar and M. B. Wise, Cambridge Monogr. Part. Phys. Nucl. Phys. Cosmol. 10, 1 (2000).

[2] K. Hagiwara et al. [Particle Data Group Collaboration], Phys. Rev. D 66, 010001 (2002).

[3] B. Aubert et al. (BABAR Collaboration), " Measurement of $\left|V_{c b}\right|$ using Lepton Energy and Hadronic Mass Moments in Semileptonic B Decays." (2003), to be submitted to Phys. Rev. Lett.

[4] S. Hashimoto et al., Phys. Rev. D 66, (2002) 014503.

[5] I. Caprini, L. Lellouch, M. Neubert, Nucl. Phys. B 530 (1998) 153; C. G. Boyd, B. Grinstein, R. F. Lebed, Phys. Rev. D 56 (1997) 6895.

[6] R.A. Briere et al. [CLEO Collaboration], Phys. Rev. Lett 89 (2002) 081803; N.E. Adam et al. [CLEO Collaboration], Phys. Rev. D 67 (2003) 032001. 